\title{
Scheme of high-resolution identification and selection of secondary ions for mass measurements with the Rare-RI Ring
}

\author{
Zhuang Ge $\mathrm{Ge}^{1,2}$ (D) . Tomohiro Uesaka ${ }^{3}$. Sarah Naimi ${ }^{3}$. Daisuke $\mathrm{Nagae}^{3}$. \\ Yasushi Abe ${ }^{3}$. Syunichiro Omika ${ }^{4}$. Fumi Suzaki ${ }^{3}$. Takayuki Yamaguchi ${ }^{4}$. \\ Yoshitaka Yamaguchi ${ }^{3}$. Masanori Wakusugi ${ }^{3} \cdot{\mathrm{Hongfu} \mathrm{Li}^{1,2} \cdot \mathrm{Kiyoshi} \text { Wakayama }}^{4}$. \\ Hiroki Arakawa ${ }^{4} \cdot K_{\text {Kumi Inomata }}{ }^{*}$ Kunimitsu Nishimuro ${ }^{4} \cdot$ Takaaki Kobayashi $^{4}$. \\ Akira Ozawa $^{5}$. Shun Hosoi ${ }^{4}$. Yasuto Inada ${ }^{4}$. Shinji Suzuki ${ }^{5}$. Tetsuaki Moriguchi ${ }^{5}$. \\ Momo Mukai ${ }^{5}$. Masamichi Amano ${ }^{5}$. Daiki Kamioka ${ }^{5}$. Yasushige Yano ${ }^{3}$
}

Published online: 20 August 2019

(C) The Author(s) 2019

\begin{abstract}
To achieve high-resolution selection and separation of mono-isotopic beams through projectile fragmentation or in-flight fission and to efficiently transport the secondary beams to the Rare-RI Ring (R3) for mass determination, the BigRIPS separator combined with the High-resolution Achromatic (HA) beam-line of SHARAQ is served as a two-stage separator. The first stage from F0 to F2 is used for separation of the nuclei of interest through a $\mathrm{B} \rho-\Delta \mathrm{E}-\mathrm{B} \rho$ method and the second stage from $\mathrm{F} 3$ to $\mathrm{S} 0$ is employed for identification of the beam with a $\mathrm{B} \rho-\Delta \mathrm{E}-\mathrm{TOF}$ method. With this scheme, all the secondary ions can be well separated on an event by event basis and be utilized for mass measurements by two complementary methods: the conventional magnetic-rigidity-time-of-flight (B $\rho$-TOF) method employing the BigRIPS-HA beam-line to measure exotic nuclides within $1 \mu$ s and the newly established isochronous mass spectrometry (IMS) approach via the R3 with a measurement duration less than $1 \mathrm{~ms}$. This report presents the technique advances of the BigRIPS-HASHARAQ-R3 as a separator/spectrometer and give a brief description of its application to the complementary TOF methods for mass measurements at RIBF.
\end{abstract}

Keywords Mass measurements · Selection · Identification · Isochronous mass spectrometry $\cdot \mathrm{B} \rho$-TOF

This article is part of the Topical Collection on Proceedings of the 7th International Conference on Trapped Charged Particles and Fundamental Physics (TCP 2018), Traverse City, Michigan, USA, 30 September-5 October 2018

Edited by Ryan Ringle, Stefan Schwarz, Alain Lapierre, Oscar Naviliat-Cuncic, Jaideep Singh and Georg Bollen

Zhuang Ge

gezhuang@impcas.ac.cn

Extended author information available on the last page of the article. 


\section{Introduction}

Since 2007, Radioactive Ion beam factory (RIBF) [1], at RIKEN Nishina center in Japan, one of the new generation facilities, has been in operation to enhance RI beam intensities for advancing experimental studies on the nuclear chart as well as to explore nuclear astrophysical processes. The RIBF accelerator complex, consisting of linacs and cyclotrons, can accelerate various kinds of heavy ions (from proton to uranium) up to $345 \mathrm{MeV} /$ nucleon, and the goal beam intensity is as high as $1 \mathrm{p} \mu \mathrm{A}$, corresponding to $6.24 \times 10^{12}$ particles/s. Since the commissioning, a variety of radioactive isotope (RI) beams has been produced at the superconducting in-flight separator BigRIPS by using in-flight fission of a ${ }^{238} \mathrm{U}$ beam as well as projectile fragmentation of several heavy ion beams such as ${ }^{18} \mathrm{O},{ }^{48} \mathrm{Ca},{ }^{70} \mathrm{Zn}$, ${ }^{78,86} \mathrm{Kr}$, and ${ }^{124} \mathrm{Xe}$.

Strong interest in fast, high-accuracy and high-precision mass measurements for exotic nuclides due to their importance in nuclear astrophysics and nuclear structure studies, has triggered the development of a various of techniques for mass measurement around the world [2-4]. An isochronous mass spectrometry (IMS) using a newly constructed storage ring named the 'Rare-RI ring' (R3) [5, 6] has been implemented at the RIKEN Nishina Center to determine the masses of short-lived rare nuclei with a relative precision of the order of $10^{-6}$. A combination of the High-resolution Achromatic (HA) beam-line (updated to be newly constructed OEDO beam-line in the year 2017), SHARAQ spectrometer [7], injection-line of R3, the R3 mass spectrometer to the BigRIPS separator makes the mass measurements by two complementary TOF (B $\rho$-TOF $[8,9]$ and IMS $[5,10])$ methods possible in one experimental setup. To realize high-resolution and high-accuracy mass measurements by the TOF methods, a scheme of high-resolution particle identification and selection of the secondary beams is employed.

The scheme of high-resolution identification and selection of secondary beams with the BigRIPS-HA beam-line for mass measurements is presented in the following section. In Section 3, the novel scheme of mass measurements with two complementary TOF methods are briefly discussed and very preliminary results of experiments for testing the complementary TOF methods for mass measurements at RIBF are demonstrated.

\section{High-resolution identification and selection of secondary beams}

A schematic view of the BigRIPS-HA-SHARAQ-R3 is shown in Fig. 1. The BigRIPSHA beam-line is primarily a powerful separator, but represents simultaneously also a highresolution spectrometer.

A thin ${ }^{9} \mathrm{Be}$ target is usually employed as the production target located at F0 of the BigRIPS separator and was bombarded by a primary beam such as ${ }^{238} \mathrm{U}^{86+}$ and ${ }^{48} \mathrm{Ca}^{20+}$ from SRC [4]. The produced secondary RIs include not only the species of interest which usually have very small production rates, but also lots of high-rate contaminations from the projectile fragmentation/fission reactions. The BigRIPS separator combined with Highresolution Achromatic beam-line of SHARAQ is served as a two-stage separator: the first stage from F0 to F2 (including four Superconducting Triplet Quadrupoles STQ1-4 and two dipoles D1-D2) is used for separation of the nuclei of interest through a $\mathrm{B} \rho-\Delta \mathrm{E}-\mathrm{B} \rho$ selection and the second stage from $\mathrm{F} 3$ to $\mathrm{S} 0$ for identification of the beam with a $\mathrm{B} \rho-\Delta \mathrm{E}-\mathrm{TOF}$ method. The second stage is also used to deduce velocity of RIs and momentum dispersion event by event. 


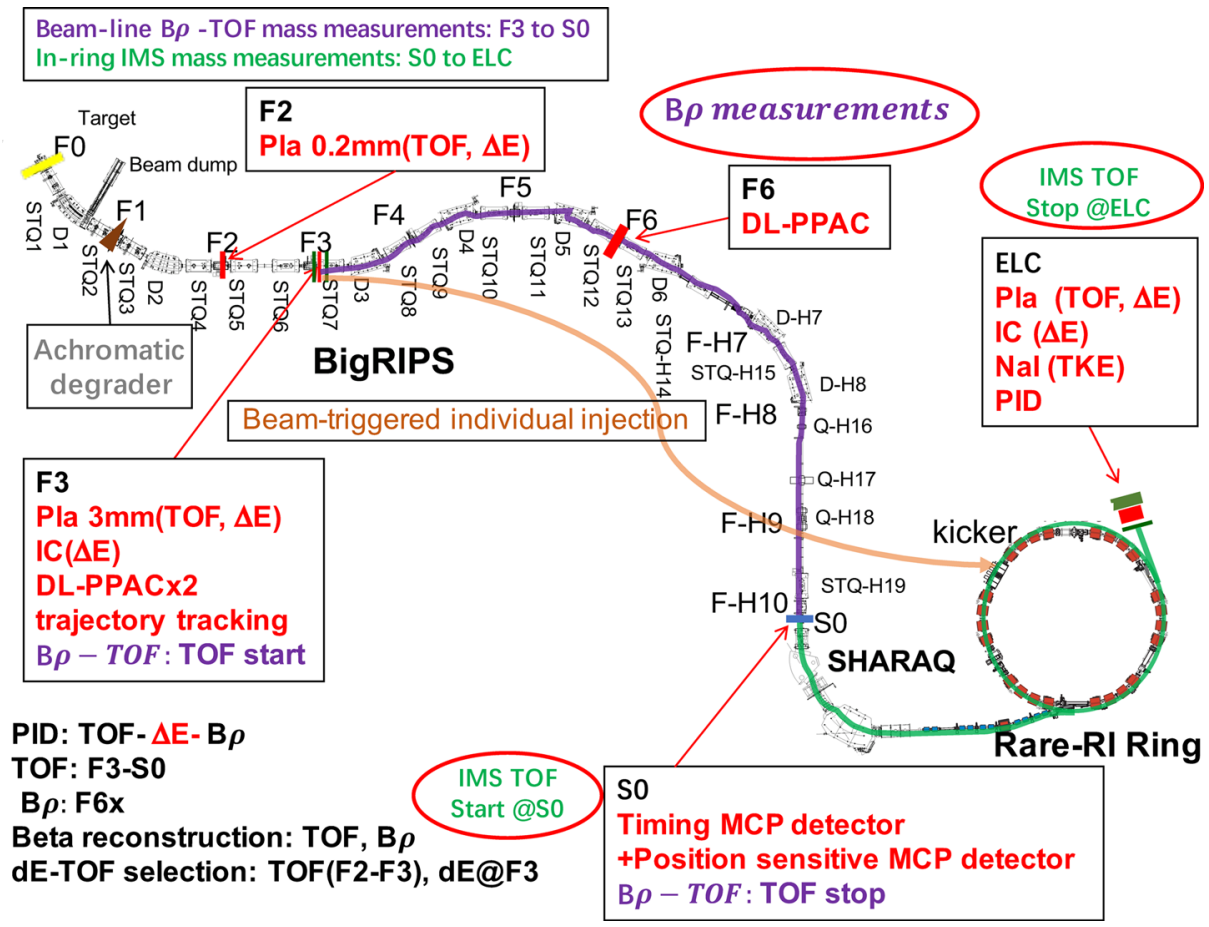

Fig. 1 A typical experimental setup for in-flight fragmentation/fission experiment at RIBF performing the R3 as an Isochronous Mass Spectrometer (IMS) and the BigRIPS-HA beam-line as a B $\rho$-TOF Mass Spectrometer

The trajectory of an ion in magnetic field $B$ depends on its mass number $A$, charge $Q=Z e$ ( $Z$ is proton number and $e$ is the charge of electron) and its momentum $P$ can be described by the equation:

$$
B \rho=\frac{P}{Q}=\frac{m v \gamma}{Q}=\frac{A}{Z} \frac{c m_{\mu}}{e} \gamma \beta,
$$

where $m_{\mu} \approx 931.494 \mathrm{MeV} / \mathrm{c}^{2}$ is the atomic mass unit, $c$ the speed of light, $\rho$ the radius of curvature, velocity of the ion relative to the speed of light $\beta=v / c$ and relativistic Lorentz factor $\gamma=\left(1-\beta^{2}\right)^{-1 / 2}$. The secondary nuclei are produced by fragmentation at F0, and the velocity is nearly constant. The selection in $B \rho$ with the first dipole D1 is then equivalent to a selection in $A / Z$. The selected nuclei by $\mathrm{D} 1$ are then transported to the $\mathrm{F} 1$ dispersive focus with the achromatic wedge-shaped degrader whose thickness varies with the horizontal plane position. The momentum and energy lost by a nucleus $(A, Z)$ in the degrader material are approximately:

$$
\Delta P \propto \frac{Z}{v}, \Delta E \propto \frac{A Z^{2}}{E} \propto\left(\frac{Z}{v}\right)^{2} .
$$

The dipole D2 with a constant magnetic field is utilized to center the nuclei of interest and disperse the nuclei possessing a different charge of $Z$ to slits in the F2 focus to stop them. Thus, nuclei with different $A / Z$ and $Z$ are separated by the momentum achromatic separator. 

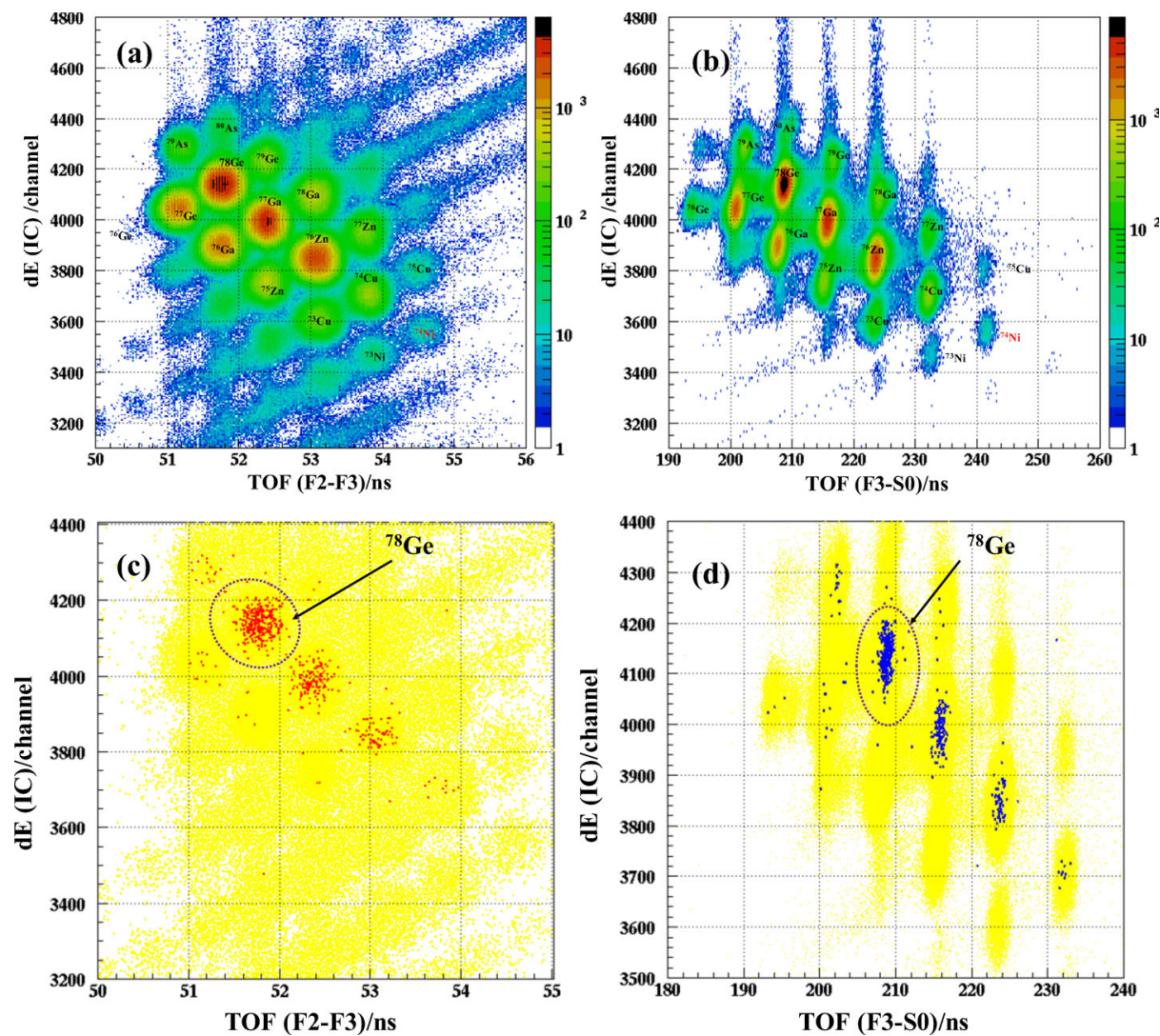

Fig. 2 a $\Delta \mathrm{E}$ (IC at F3) -TOF (F2 to F3) 2D histogram b $\Delta \mathrm{E}$ (IC at F3) -TOF (F3 to S0) 2D histogram c 2D correlation histogram of TOF- $\triangle \mathrm{E}$ (TOF of F2 to F3 and $\Delta \mathrm{E}$ at F3 IC) and red points represent the extracted ions from R3. The yellow backgrounds are all the ions that arrive at F3 focal plane. (d) Identified particles in $2 \mathrm{D}$ correlation histogram of TOF- $\triangle \mathrm{E}$ (TOF from $\mathrm{F} 3$ to $\mathrm{S} 0$ and $\Delta \mathrm{E}$ at $\mathrm{F} 3 \mathrm{IC}$ ) for experimental run of IMS mass measurements, in which the blue corresponds to the extracted ions and the yellow are all the ions that arrive at S0 focal plane

In order to inject RIs of interest into the R3 event by event, a self-triggered individual injection technique is developed [6]. When a produced secondary particle passes through the timing detector (plastic scintillator) at F3 of the BigRIPS, a trigger signal is generated. The maximum injection repetition frequency of kicker system is $100 \mathrm{~Hz}$ which limits the rate of trigger events that could excite the kicker magnets to inject themselves into R3. To separate and inject the RIs of interest efficiently to R3, a technique by the combination of the trigger signal of only the RIs of interest at F3 with the radio frequency (RF) timing signal for accelerators is developed (details can be found in [11]). During the beam tuning by this method, $\triangle \mathrm{E}$-TOF method is utilized for particle identification (PID) from F2 to F3. Typical $2 \mathrm{D}$ histograms of $\Delta \mathrm{E}-\mathrm{TOF}$ ( $\Delta \mathrm{E}$ by F3 Ionization Chamber (IC) and TOF from F2 to F3 by plastic scintillators) for PID, as shown in Fig. $2 \mathrm{a}$ and c, can be used for online beam tuning and offline analysis as well.

The identification of the resulting secondary cocktail beams on an event by event basis is performed in the second part of the BigRIPS-HA beam-line (F3-S0). The 
doubly-achromatic high-resolution beam-line is composed of ten Superconducting Triplet Quadrupoles (STQ7-13, STQ-H14-H15,STQ-H19), four normal-conducting quadrupoles (Q-H16-17, Q-H18a and Q-H18b), and six dipoles (D3-D6, D-H7-8), with the F4, F5, F6 as momentum dispersive planes and F3, S0 as doubly-achromatic foci. An achromatic system normally has the best performance for spatial separation of mono-isotopic beams. The nuclei of interest are transmitted up to the doubly-achromatic S0 focal plane.

The particle identification of the secondary cocktail beams is achieved by the $\mathrm{B} \rho-\Delta \mathrm{E}-$ TOF method in the BigRIPS-HA spectrometer, which ensures tagging of every particle delivered in the form of a cocktail beam from projectile fragments or in-flight fission. Gamma-ray detector for isomer tagging at F7 focus is used to ensure the PID.

The PID at the secondary stage from F3 to S0 provided the atomic number $Z$ and massto-charge ratio $A / Q$ of a fragment by measuring the energy loss, magnetic rigidity and time-of-flight ( $\Delta \mathrm{E}-\mathrm{B} \rho$-TOF) with the corresponding beam-line detectors. Typical $\Delta \mathrm{E}-\mathrm{TOF}$ 2D histograms for PID from F3 focus to S0 focus are shown in Fig. 2b and d.

The TOF between two foci F3 and S0 is measured by the plastic scintillator at F3 and the foil-MCP detector [16] at $\mathrm{S} 0$ as shown in Fig. 1: $T O F=L /(\beta c)$, can be used for $\beta$ measurements.

From $B \rho=\frac{P}{Q}=\frac{m v \gamma}{Q}=\frac{A}{Q} \frac{c m_{\mu}}{e} \gamma \beta$, we can get:

$$
\frac{A}{Q}=\frac{B \rho}{\beta \gamma} \frac{e}{m_{\mu} c} .
$$

The atomic number $Z$ of a heavy ion was deduced based on the energy loss of the charged particle in the F3 IC. The energy loss of a heavy ion in an unit thickness of material can be described by the Bethe-Block formula as [4]:

$$
-\frac{d E}{d X}=\frac{4 \pi Z^{2} e^{4} N Z_{m}}{m_{e} c^{2} \beta^{2}}\left[\ln \frac{m_{e} c^{2} \beta^{2}}{I}-\ln \left(1-\beta^{2}\right)-\beta^{2}\right],
$$

and then the formula calculating the atomic number $\mathrm{Z}$ can be written as:

$$
Z=a_{1} \beta \sqrt{\frac{\Delta E}{\ln \left(\frac{2 m_{e} c^{2} \beta^{2}}{I}\right)-\ln \left(1-\beta^{2}\right)-\beta^{2}}}+a_{2},
$$

where $a_{1}$ and $a_{2}$ are parameters to be calibrated from the experiment to reconstruct the $\mathrm{Z}$ value for each ion. $I$ is the mean excitation potential of the counter gas; $Z_{m}$, atomic number of the counter gas; $Z$, atomic number of heavy ion; $m_{e}=0.511 \mathrm{MeV} / \mathrm{c}^{2}$, electron mass. Two reconstructed $A / Q-Z 2 \mathrm{D}$ histograms for PID of in-flight fission fragments from primary beam of ${ }^{238} \mathrm{U}^{92+}$ and projectile fragments from primary beam of ${ }^{48} \mathrm{Ca}^{20+}$ are shown in Fig. 3a and b, respectively. As shown in Fig. 3, due to the excellent resolving power of the BigRIPS-HA beam-line, the resolution of $A / Z$ and $Z$ is good enough to resolve all the highly charged RIs.

With the establishment of the scheme of high-resolution separation, identification and selection of secondary RIs at the BigRIPS-HA beam-line, we could individually inject the ions of interest with a limited rate of $100 \mathrm{~Hz}$ to the R3 which has a relative momentum acceptance $\pm 0.3 \%$ for IMS mass measurements. Meanwhile, some of the nuclei which are not injected into R3 but accepted by the high-resolution BigRIPS-HA beam-line within a relative momentum acceptance $\pm 0.5 \%$ are also of interest, and the reachable ones will be measured simultaneously via the $\mathrm{B} \rho$-TOF method. Figure $3 \mathrm{c}$ and $\mathrm{d}$ schematically show the acceptance difference of the beam-line and the R3 by comparing the counts of ions arriving 

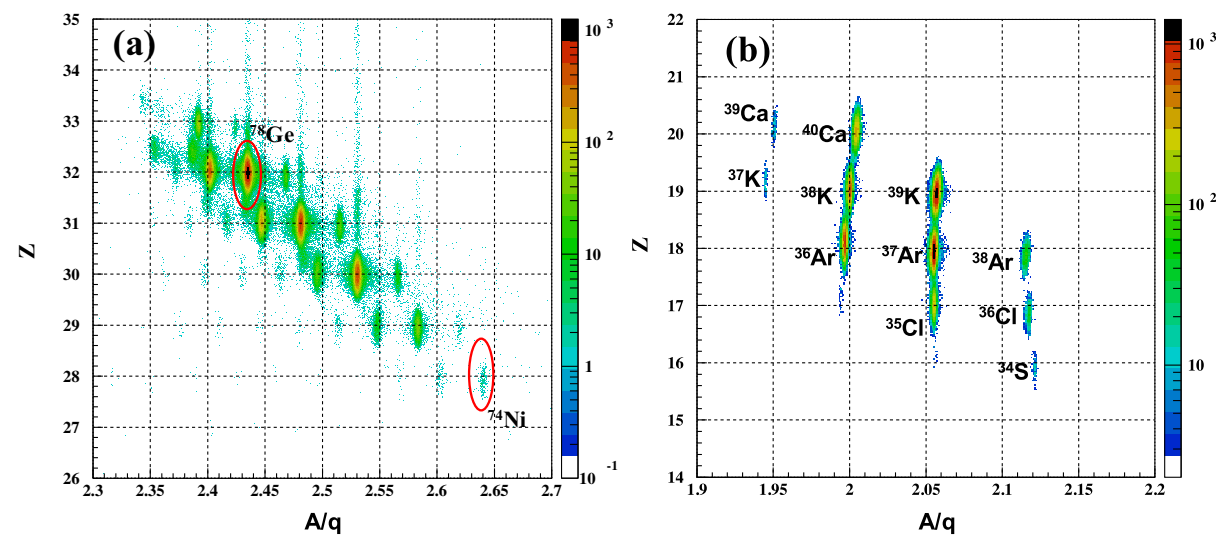

Fig. 3 a 2D correlation histogram of $A / q$ and $Z$ for particle identification of in-flight fission fragments with a primary beam of ${ }^{238} \mathrm{U}$. b $2 \mathrm{D}$ correlation histogram of $A / q$ and $Z$ for particle identification of in-flight projectile fragments with a primary beam of ${ }^{48} \mathrm{Ca}$

at $\mathrm{F} 3 / \mathrm{S} 0$ and the counts of those extracted from R3. The high-resolution identification of secondary RIs will also benefit the subsequent data analysis processes for mass deduction. Especially, the identification of $\mathrm{N}=\mathrm{Z}$ nuclei with very close $\mathrm{m} / \mathrm{q}$ values, which can not be realized by other in-ring TOF techniques for mass measurements developed at CSRe/IMP and ESR/GSI [10], could be easily carried out with the scheme described above.

\section{A novel scheme of mass measurements by complementary Time-of-Flight methods}

\subsection{Mass measurements by IMS method}

The basic principle for storage ring mass spectrometry describing the relationship between mass-over-charge ratio $(\mathrm{m} / \mathrm{q})$ and revolution period $(T)$ or revolution frequency $(f)$ can be quantitatively expressed in first order approximation [10]:

$$
\frac{d T}{T}=-\frac{d f}{f}=\frac{1}{\gamma_{t}^{2}} \frac{d(m / q)}{m / q}-\left(1-\frac{\gamma^{2}}{\gamma_{t}^{2}}\right) \frac{d v}{v}=\frac{1}{\gamma^{2}} \frac{d(m / q)}{m / q}+\left(\frac{1}{\gamma_{t}^{2}}-\frac{1}{\gamma^{2}}\right) \frac{d(B \rho)}{B \rho},
$$

where $\gamma_{t}$ is the so-called transition energy of the ring.

In order to determine the $m / q$ values from the measured revolution times of the ions, the second term of (1) right-hand side has to be made negligibly small: $\left(1-\frac{\gamma^{2}}{\gamma_{t}^{2}}\right) \frac{d v}{v} \rightarrow 0$. Based on this principle, two methods, namely Schottky (SMS) with $\frac{d v}{v} \rightarrow 0$ by cooling the ions and isochronous mass spectrometry (IMS) with an isochronous optical setting $(\gamma \rightarrow$ $\gamma_{t}$ ), have been developed for mass measurements.

In conventional IMS method at GSI and CSRe with a typical mass accuracy of $10^{-6}-$ $10^{-7}$, one TOF detector is installed in the ring for particle identification by measuring the in-ring TOF spectrum and deduce mass without extra $B \rho$ or $\beta$ measurements of ions. Only one species of nucleus can fulfill isochronous condition $\gamma=\gamma_{t}$ in one setting of the beamline and optics of storage. To decrease the spread of revolution period of stored ions in 
non-isochronous condition, additional magnetic rigidity or velocity measurements of the ions should be precisely carried out. By employing this additional information, the mass resolving power of IMS will be significantly improved.

The R3 is dedicatedly designed to measure the exotic nuclei in IMS mode with additional velocity or magnetic rigidity measurement for mass determination, which depends on the hypothesis that ions with the same magnetic rigidity $(B \rho)$ will move around the same close orbit, regardless of their species. Therefore, the correction of the in-ring revolution time by $B \rho$ (equivalent to velocity for a certain close orbit) measurements can be carried out. The mass-to-charge ratio $m_{1} / q_{1}$ for the nucleus with unknown mass could be expressed as [5]:

$$
\frac{m_{1}}{q_{1}}=\frac{m_{0}}{q_{0}} \frac{T_{1}}{T_{0}} \sqrt{\frac{1-\beta_{1}^{2}}{1-\left(\frac{T_{1}}{T_{0}}\right)^{2} \beta_{1}^{2}}}=\frac{m_{0}}{q_{0}} \frac{T_{1}}{T_{0}} \sqrt{\frac{1-\left(\frac{T_{0}}{T_{1}}\right)^{2}}{\left(\frac{m_{0}}{q_{0}} c\left((B \rho)_{0}\right)^{-1}\right)^{2}}+1}
$$

When a particle of interest with a mass-to-charge ratio $m_{1} / q_{1}$ has the same $B \rho$ as that of a reference particle with a mass-to-charge ratio $m_{0} / q_{0}$, the flight path length of these particles become identical in the isochronous storage ring. $m_{1} / q_{1}$ can be deduced from the ratio $m_{0} / q_{0}$ of the reference nucleus with the revolution time $(T)$ and velocity $(\beta)$ /momentum $(B \rho)$ measurements of all ions. The parameter $K=\sqrt{\frac{1-\beta_{1}^{2}}{1-\left(\frac{T_{1}}{T_{0}}\right)^{2} \beta_{1}{ }^{2}}}$ and $P=\sqrt{\frac{1-\left(\frac{T_{0}}{T_{1}}\right)^{2}}{\left(\frac{m_{0}}{q_{0}} c\left((B \rho)_{0}\right)^{-1}\right)^{2}}+1}$ are defined as velocity correction factor and momentum correction factor, respectively.

\subsubsection{Test of beam-lines in conjunction with the Rare-RI Ring as IMS}

The experimental setup utilizing the R3 as an IMS is demonstrated in Fig. 1. When a produced secondary particle passes through the timing detector (Plastic scintillator) at F3 focal plane of the BigRIPS separator, a trigger signal will be generated and transmitted to a fast-kicker system via a high speed coaxial tube. kicker magnets are then immediately excited by thyratrons. In the meanwhile, the particle that arrives at the entrance of the ring is injected into the central orbit of the ring using septum and kicker magnets. After the particle revolves in the ring about $700 \mu s$, it will be extracted using another septum and the same kicker magnets. The start of total TOF for IMS is set at doubly-achromatic focus S0 of SHARAQ beam-line by using foil-MCP detector, and stop of total TOF is measured at the extraction-line course (ELC) of R3 by using a plastic scintillator. In addition, the extracted ion can be identified by $\Delta \mathrm{E}-\mathrm{E}$ detectors (Ionization Chamber and $\mathrm{NaI}$ ) located at the end of extraction line. Thus we can combine the beam-lines and R3 to perform a $\mathrm{B} \rho$-TOF- $\Delta \mathrm{E}-$ $\mathrm{E}$ method for PID. The $\beta$ or $B \rho$ measurement is necessary to correct the revolution time of non-isochronous condition particles if extracted from R3. The measurement of $B \rho$ at a dispersive focal plane F6 by a delay-line parallel plate avalanche counters (PPAC) with the TOF measured from F3 to S0 can be employed to deduce the $\beta$ (velocity) of each RI. In addition to the short measurement time $(\sim 700 \mu s)$, this method enables us to measure the mass of even one ion which is extracted with the same setting as the reference nuclei. The extraction efficiency for R3 in this test is about $0.14 \%[12,13]$. In a most recent experiment, this value of extraction efficiency get improved by a factor of $\sim 14(1.9 \%)$ and our goal 

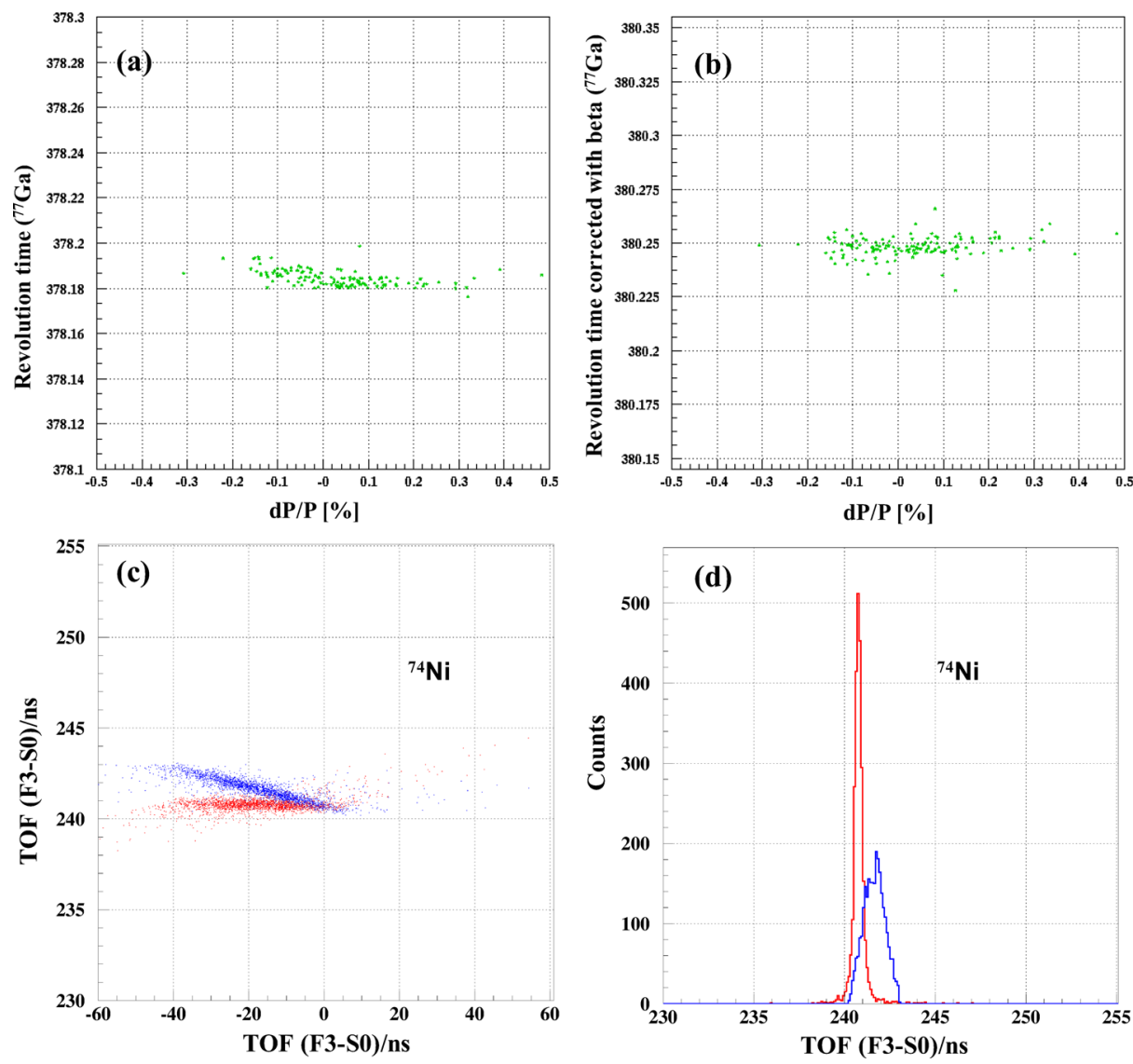

Fig. 4 a Spectrum of the measured revolution time of fully stripped ${ }^{77} \mathrm{Ga}$ as a function of the relative momentum dispersion. b Spectrum of the velocity-corrected revolution time (gated ${ }^{77} \mathrm{Ga}$ ) as a function of the relative momentum dispersion. c Relation between TOF (F3-S0) and $\mathrm{X}_{F 6}$ for gated ${ }^{74} \mathrm{Ni}$. Blue is the relation before correction and red illustrates the one after correction. d TOF (F3-S0) spectrum before (in blue) magnetic rigidity correction and the one after (in red) correction

efficiency is $20 \%$ [12, 13]. In Fig. 4a, the 2D spectrum of in-ring revolution time as a function of relative momentum dispersion of the extracted ions of ${ }^{77} \mathrm{Ga}$ employing a PID gate is illustrated. After correcting the in-ring revolution time with the velocity correction factor $\mathrm{K}$, the $2 \mathrm{D}$ spectrum of velocity-corrected in-ring revolution time of fully stripped ${ }^{77} \mathrm{Ga}$ as a function of the relative momentum dispersion is displayed in Fig. 4b. The momentum dependence of the revolution time is removed by employing the velocity correction method. Only very preliminary results are shown in Fig. $4 \mathrm{a}$ and $\mathrm{b}$ for this test. The final results of the IMS test will be published somewhere else [14].

\subsection{Mass measurements by B $\rho$-TOF method}

The $\mathrm{B} \rho$-TOF technique $[8,9]$ for mass measurements of exotic nuclei is based on measurement of the magnetic rigidity and the corresponding time-of-flight of the ion:

$$
B \rho=\frac{m \gamma}{q} \frac{L}{T O F} .
$$


The mass-to-charge ratio $m / q$ is derived from (8):

$$
m / q=\frac{B \rho}{\gamma L / T O F}=B \rho \sqrt{\left(\frac{T O F}{L}\right)^{2}-\left(\frac{1}{c}\right)^{2}},
$$

where TOF can usually be determined with very high precision, while the measurements of magnetic rigidity $\mathrm{B} \rho$ and flight length $L$ would severely limit the precision of the resultant mass. Thus in practice nuclei with well-known masses are measured along with nuclei of unknown mass in order to calibrate the relationship between TOF, rigidity, and mass. Consequently one yields a simple relationship between TOF and mass of the nuclei of interest. The mass uncertainty can be deduced from (9):

$$
\frac{\delta m}{m}=\frac{\delta(B \rho)}{B \rho}+\frac{1}{1-\left(\frac{1}{c} \frac{L}{T O F}\right)^{2}}\left(\frac{\delta(T O F)}{T O F}-\frac{\delta L}{L}\right) .
$$

The mass resolving power of this technique depend on the detector (TOF and position measurement) resolutions and the momentum resolution of the beam-line. $\mathrm{B} \rho$-TOF mass measurement with a typical TOF $\sim 1 \mu$ s provides mass data with an accuracy from $10^{-4}$ up to the level of $10^{-6}$ which is usually depending on the statistics and allow simultaneous measurements of many nuclides, reference isotopes as well as isotopes of interest.

\subsubsection{Test of BigRIPS and High-resolution beam-line as $\mathrm{B} \rho$-TOF Mass spectrometer}

Figure 1 also includes the setup for $\mathrm{B} \rho$-TOF mass measurements in the same experimental run in parallel and complementarily. The TOF is measured from F3 with a plastic scintillator as TOF start to S0 with a foil-MCP detector as TOF stop. The beam position is measured at $\mathrm{F} 6$ for $\mathrm{B} \rho$ determination, and at $\mathrm{F} 3$ with PPAC detectors for emittance measurement. The timing resolution of the foil-MCP detector is $\sim 130 \mathrm{ps}$, and the timing resolution of $\mathrm{F} 3$ plastic scintillator is about $20 \mathrm{ps}$ in this test experiment. Because the absolute path length of the beam line and the magnetic field of the dipoles can not be measured with the required precision, the relationship between TOF and $\mathrm{m} / \mathrm{q}$ has to be calibrated with reference nuclei with precisely known masses. The fitted slop information from the TOF and $\mathrm{X}_{F 6}$ correlation histogram of each nucleus are used for the TOF correction. As shown in Fig. 4c, the blue scattering points corresponds to the original TOF versus $\mathrm{X}_{F 6}$ of the ions of ${ }^{74} \mathrm{Ni}$, and the red indicates the magnetic rigidity corrected TOF distributions as a function of the $\mathrm{X}_{F 6}$. The corrected TOF in red shows a momentum independent distribution. The projected original TOF distribution and final rigidity-corrected TOF distribution for ${ }^{74} \mathrm{Ni}$ are both very close to Gaussian function as shown in blue and red in Fig. 4d. The gaussian function fitted original TOF distribution (the standard deviation $\sigma$ ) for ${ }^{74} \mathrm{Ni}$ is $573.4 \mathrm{ps}$. After magnetic rigidity correction, the fitted TOF distribution (the standard deviation $\sigma$ ) of ${ }^{74} \mathrm{Ni}$ is $\sim 200 \mathrm{ps}$. This uncertainty $\sigma$ mainly comes from the low resolution of the timing detector at S0. With a total TOF from $\mathrm{F} 3$ to $\mathrm{S} 0$ about $550 \mathrm{~ns}$, the resolving power of the TOF measurement with the preliminary correction by magnetic rigidity reached $3.6 \times 10^{-4}$. The subsequent analysis is going on: corrections of the TOF by other measured parameters such as emittance, trajectory and identified proton number $Z$ of the corresponding nuclei.

The transmission efficiency of $\sim 80 \%$ up to S0 focus is achieved for this test. As shown in Fig. $2 \mathrm{~d}$, the nuclei with a relative momentum acceptance $\pm 0.5 \%$ is accumulated by the beam-line, while the nuclei of mainly one isotonic chain $\mathrm{N}=46$ with a relative momentum acceptance $\pm 0.3 \%$ is accepted by the R3 [15]. It is essential to measure the masses of nuclei of interest within the acceptance of the high-resolution beam-line but out of the acceptance 
of $\mathrm{R} 3$ with the $\mathrm{B} \rho$-TOF method. The foil-MCP detector at $\mathrm{S} 0$ is optimized to be $\sim 40 \mathrm{ps}$ [16] and is ready to be utilized for the future mass-measurement experiment at RIBF.

\section{Summary}

A scheme of high-resolution particle identification and selection of the secondary beams at RIBF for two complementary TOF mass measurements methods has been developed. The scheme has been successfully studied in online experiments of projectile fragmentation (primary beam of ${ }^{48} \mathrm{Ca}$ ) and in-flight fission (primary beam of ${ }^{238} \mathrm{U}$ ) with the R3 as IMS in RIBF. This scheme will be utilized for the identification and selection of $\mathrm{N}=\mathrm{Z}$ nuclei for mass measurements by IMS method at the R3, and the nuclei nearby will be measured by $\mathrm{B} \rho$-TOF approach via the high-resolution beam-line with relatively lager acceptance in the future.

Open Access This article is distributed under the terms of the Creative Commons Attribution 4.0 International License (http://creativecommons.org/licenses/by/4.0/), which permits unrestricted use, distribution, and reproduction in any medium, provided you give appropriate credit to the original author(s) and the source, provide a link to the Creative Commons license, and indicate if changes were made.

\section{References}

1. Yano, Y:: The RIKEN RI Beam Factory Project: A status report. Nucl. Instrum. Methods Phys. Res. B 261, 1009-1013 (2007)

2. Lunney, D., Pearson, J.M., Thibault, C.: Recent trends in the determination of nuclear masses. Rev. Modern Phys. 75, 1021-1082 (2003)

3. Blaum, K.: High-accuracy mass spectrometry with stored ions. Phys. Rep. 425, 1-78 (2006)

4. Fukuda, N., Kubo, T., Ohnishi, T., Inabe, N., Takeda, H., Kameda, D., Suzuki, H.: Identification and Separation of Radioactive Isotope Beams by the bigRIPS Separator at the RIKEN RI Beam Factory. Nucl. Instrum. Methods Phys. Res. B 317, 323 (2013)

5. Ozawa, A., Uesaka, T., Wakasugi, M.: The Rare-RI Ring collaboration: the Rare-RI Ring. Prog. Theor. Exp. Phys. 2012, 03 C009 (2012)

6. Yamaguchi, Y., Wakasugi, M., Uesaka, T., Ozawa, A., Abe, Y., Fujinawa, T., Kase, M., Komiyama, M., Kubo, T., Kumagai, K., Maie, T., Nagae, D., Ohnishi, J., Suzaki, F., Tokuchi, A., Watanabe, Y., Yoshida, K., Yamada, K., Yamaguchi, T., Yamasawa, H., Yanagisawa, Y., Zenihiro, J., Yano, Y.: Construction of rare-RI ring at RIKEN RI Beam Factory. Nucl. Instrum. Methods Phys. Res. B 317, 629-635 (2013)

7. Uesaka, T., Shimoura, S., Sakai, H.: the SHARAQ Collaboration: The SHARAQ spectrometer. Prog. Theor. Exp. Phys. 2012, $03 \mathrm{C} 007$ (2012)

8. Meisel, Z., George, S.: Time-of-flight mass spectrometry of very exotic systems. Int. J. Mass Spectrom. 349-350, 145-150 (2013)

9. Michimasa, S., Kobayashi, M., Kiyokawa, Y., Ota, S., Ahn, D.S., Baba, H., Berg, G.P.A., Dozono, M., Fukuda, N., Furuno, T., Ideguchi, E., Inabe, N., Kawabata, T., Kawase, S., Kisamori, K., Kobayashi, K., Kubo, T., Kubota, Y., Lee, C.S., Matsushita, M., Miya, H., Mizukami, A., Nagakura, H., Nishimura, D., Oikawa, H., Sakai, H., Shimizu, Y., Stolz, A., Suzuki, H., Takaki, M., Takeda, H., Takeuchi, S., Tokieda, H., Uesaka, T., Yako, K., Yamaguchi, Y., Yanagisawa, Y., Yokoyama, R., Yoshida, K., Shimoura, S.: Phys. Rev. Lett. 121, 022506 (2018)

10. Zhang, Y.H., Litvinov, Yu.A., Uesaka, T., Xu, H.S.: Storage ring mass spectrometry for nuclear structure and astrophysics research. Phys. Scr. 91, 073002 (2016)

11. Abe, Y.: The Rare-RI Ring Collaboration: RIKEN Accelerator Progress Report 50,186 (2017)

12. Yamaguchi, Y.: The Rare-RI Ring Collaboration: RIKEN Accelerator Progress Report 51,165 (2018)

13. Naimi, S.: The Rare-RI Ring Collaboration: RIKEN Accelerator Progress Report 51,166 (2018)

14. Yamaguchi, Y.: the Rare-RI Ring Collaboration, in preparation

15. Nagae, D.: the Rare-RI Ring Collaboration: RIKEN Accelerator Progress Report 51,168 (2018)

16. Ge, Z.: the Rare-RI Ring Collaboration: RIKEN Accelerator Progress Report 51,152 (2018) 
Publisher's note Springer Nature remains neutral with regard to jurisdictional claims in published maps and institutional affiliations.

\section{Affiliations}

Zhuang $\mathrm{Ge}^{1,2} \mathbb{1}$. Tomohiro Uesaka $^{3} \cdot$ Sarah Naimi ${ }^{3}$. Daisuke Nagae ${ }^{3}$. Yasushi Abe ${ }^{3}$. Syunichiro Omika ${ }^{4}$. Fumi Suzaki ${ }^{3}$. Takayuki Yamaguchi ${ }^{4}$.

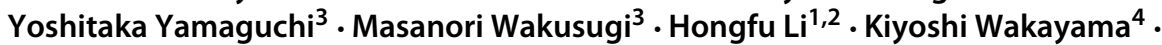
Hiroki Arakawa ${ }^{4} \cdot$ Kumi Inomata $^{4} \cdot$ Kunimitsu Nishimuro $^{4} \cdot$ Takaaki Kobayashi $^{4}$. Akira Ozawa ${ }^{5}$. Shun Hosoi ${ }^{4}$. Yasuto Inada ${ }^{4}$. Shinji Suzuki ${ }^{5}$. Tetsuaki Moriguchi ${ }^{5}$. Momo Mukai ${ }^{5}$. Masamichi Amano ${ }^{5}$. Daiki Kamioka ${ }^{5}$. Yasushige Yano ${ }^{3}$

1 Institute of Modern Physics, Chinese Academy of Sciences, Lanzhou, 730000, People's Republic of China

2 University of Chinese Academy of Sciences, Beijing, 100049, People's Republic of China

3 RIKEN Nishina Center, RIKEN, 2-1 Hirosawa, Wako, Saitama 351-0198, Japan

4 Department of Physics, Saitama University, Shimo-Okubo 255, Sakura-ku Saitama-shi, 338-8570, Japan

5 Institute of Physics, University of Tsukuba, 1-1-1 Tennodai, Tsukuba, Ibaraki 305-8571, Japan 